\title{
Turmeric polyphenols: A comprehensive review
}

\author{
Muhammad Hanif Mughal* \\ Homeopathic Clinic, Islamabad, Pakistan
}

\begin{abstract}
Turmeric (curcuma longa) is a spice of yellow color and has been used to preserve food. Curcumin (diferuoyl methane) a yellow active ingredient in turmeric is a feruloylmethane homodimer consisting of a band of hydroxyl and methoxy (heptadiene with two Michael acceptors) and a b-diketone. Turmeric is capable of influencing the Akt, growth factors, NF- $\mathrm{kB}, \&$ metastatic and angiogenic pathways. It modulates gene expression in human cancer cells in a time-and concentrationdependent way. Curcumin has good therapeutic and preventive potential against several major human conditions such as cardiovascular, inflammation suppression, antimicrobial, obesity, tumorigenesis, chronic tiredness, antidepressant \& neurological function, anxiety, muscle \& bone loss, and neuropathic pain.
\end{abstract}

\section{Introduction}

Today, due to improper food preparation and lifestyle changes, almost half of the world's population suffers from chronic diseases. Unsuitable feeding habits are the main causes of hunger and death, which is the main cause of health problems. Currently, the most common for health promotion has been functional foods and dietary supplements. Multiple physiological diseases can be controlled by diets containing several biologically active ingredients. Due to these treatments, dietary supplements and functional foods are important to enhance consumer health and reduce numerous diseases such as obesity, heart disease, hyperlipidemia and cancers [1].

Scientists are working to create usable foods in modern times. We have a major health-related variable due to the presence of carotenoids and polyphenols in functional and dietary supplements. Herbs and spices contribute to a range of therapies, including diuretics, ulcers, diuretics, bronchi, tonic and toxic medicines [2].

Turmeric (Curcuma longa) belongs to the family Zingiberaceae which is native to Southeast Asian countries and is derived from turmeric roots, often used as spice in different curry products. Nutritional components in turmeric are ukonan-A, diarypeptanoids, essential oil (3-5\%), tumerin, turmeran-D (antitumorigenic), vitamin A, carotenoids and minerals. Turmeric also contains sesquiterpenes, carbohydrates, proteins, resins, and caffeic acid. Curcumin was the first isolated element of turmeric that was isolated in 1815 and a laughry and whitening determined its chemical composition in 1977 [3].

Turmeric's main component is curcumin, which is extracted by solvent extraction method from turmeric. Curcumin is stable in high temperature range and acidic state, but is volatile in alkaline environments as well as in the presence of heat. Curcumin is soluble in oil and alkali, and at acid and neutral $\mathrm{pH}$ is virtually insoluble in water. Water-soluble curcumin is prepared by incorporation of sodium dodecyl sulfate, cetylpyridinium bromide, gelatine, polysaccharides, polyethylenglycol and cyclodextrins into various surfactant micellar systems. Commercial curcumin is usually a combination of three curcuminoids: demethoxycurcumin $(19.4 \%)$, curcumin $(71.5 \%)$, and bisdemethoxycurcumin $(9.1 \%)$ and curcumin is prepared by using different solvents carbon dioxide, acetone, and ethanol from ground turmeric rhizomes [4].

\section{Chemical composition}

Considering nutritional profiling of turmeric, moisture (8.92\%), crude fiber (4.60\%), ash (2.85\%), fat (6.85\%), crude protein $(9.40 \%)$, and carbohydrate $(67.38 \%)$, respectively. The fiber has been found to clean the digestive tract of consumer by removing potential carcinogens from the body and can prevent the absorption of excessive cholesterol. Fiber also adds bulk to the food and prevents the intake of excessive starchy foods and may therefore act as guard against metabolic problems such as hypercholesterolemia and diabetes mellitus [5].

Alkaloid in turmeric plant indicates that in treating headache caused by hypertension, cold, chronic catarrh, and migraine, turmeric could be used. Turmeric plant may be beneficial for inflammation control, sex hormone enhancement, cholesterol reduction, deleterious prevention, and cytotoxins. Saponin, flavonoids and tannin have been found effective in treatment of intestinal disorder such as diarrheal condition and dysentery. Considering minerals, turmeric has phosphorus $(0.63 \%)$, potassium $(0.46 \%)$, calcium $(0.20 \%)$, and iron $(0.05 \%)$. Turmeric polyphenols have role in strong bone, blood clothing, muscle contraction and relaxation, and reducing blood pressure. For iron deficiency anemia, turmeric is recommended because the iron content in the extract can aid in the production of hemoglobin. Phytochemical composition of turmeric includes $0.4 \%$ saponin, $0.76 \%$ alkaloid, $0.03 \%$ sterol, $1.08 \%$ tannin, $0.40 \%$ flavonoid, $0.82 \%$ phytic acid, and $0.08 \%$ phenol [6].

In composition analysis of turmeric contain curcumin, essential oil (3-5\%) (which is act as antibacterial agent and anti-inflammatory agent), fat, Tumerin (protects DNA), Ukonan-A (whitened activated blood cells)), Ukonan-D (anti carcinogenic), vitamin A, carotenoids, minerals. The proximate composition of turmeric is $8.92 \%$ moisture,

${ }^{\star}$ Correspondence to: Muhammad Hanif Mughal, Homeopathic Clinic, Islamabad, Pakistan, E-mail: drmhanifmughal@gmail.com

Key words: turmeric, chemical composition, curcumin, antidiabetic, hypercholesterolemia

Received: September 19, 2019; Accepted: October 07, 2019; Published: October 11,2019 
$2.85 \%$ ash, $4.60 \%$ crude fiber, $6.85 \%$ fat, $9.40 \%$ crude protein, $67.38 \%$ carbohydrate. This suggests that it may be a good source of proteins and carbohydrates. The mineral composition of turmeric is $0.20 \%$ calcium, $0.63 \%$ phosphorus, $0.46 \%$ potassium, $0.05 \%$ iron [7]. Continuous feeding of turmeric plants is very useful for strong bone, muscle contraction and relaxation, reduction in blood pressure, reduction in blood pressure, and maintenance of hemoglobin formation. Turmeric is recommended for iron deficiency anemia. It is because the iron contained in the extract can help form hemoglobin [8].

Turmeric plants typically contain $0.76 \%$ alkaloid, $0.45 \%$ saponin, $1.08 \%$ tannin $0.03 \%$ sterol, $0.82 \%$ phytic acid, $0.40 \%$ flavonoid and $0.08 \%$ phenol. Among turmeric plants, the presence of alkaloids $(0.76$ percent) indicates that turmeric can be used to treat hypertensionrelated headaches, treat colds, and treat chronic migraine. Turmeric plants can enhance sex hormones, lower cholesterol, prevent harmful cytotoxins and reduce inflammation due to antioxidant properties of saponins, flavonoids and tannins. Turmeric plants show their antioxidant properties and can be used to treat diseases of the intestines such as diarrhea and dysentery [9].

\section{Bioavailability and stability}

The oral bioavailability of curcumin in the gastrointestinal is poor and produces hexahydro curcumin, curcumin sulphate, curcumin glucuronide, tetra hydroxyl curcumin, and dihydrocurcumin. Because of its tautomer enol structure, curcumin has low bioavailability. It provides three protons producing ions in a water solution, the enolic proton with $8.5 \mathrm{pKA}$, whereas the other two phenolic protons have a pKA value ranging from 10 to 10.5 . Curcumin has low bioavailability due to its tautomer enol structure. It gives three protons releasing ions in a water solution, the enolic proton with $8.5 \mathrm{pKA}$, while the other two phenolic protons have 10 to $10.5 \mathrm{pKA}$ value. Numerous laboratories with different results have studied the chemical degradation of curcumin by alkali due to media disparity. Previous investigations identified degradation products such as feruloyl methane and curcumin ferulic acid and also studied degradation kinetics in a $\mathrm{MeOH}$-aqueous buffer solution (phosphate buffer pH 6-9) [10].

It is also shown that curcumin decomposed at $\mathrm{pH} 7.2$ by 90 percent and at temperature $378^{\circ} \mathrm{C}$ by $0.1 \mathrm{M}$ phosphate buffer within $30 \mathrm{~min}$ and uncertainly labeled the decomposed material as trans6-(4'-hy-droxy-3'-methoxyphenyl)-2,4-dioxo-5-hexenal. Vanillin is known as a major product along with ferulic acid and feruloylmethane from the decomposed products of curcumin. Curcumin lowers lipid peroxidation and maintains a range of antioxidant enzymes, i.e. catalase, glutathione peroxidase, and dis-mutase superoxide. In addition, curcumin's therapeutic ability was prevented by enhancing glutathione levels and biosynthesis by Nrf2 from free radicals [11]. Because of its many health benefits, curcumin is gaining worldwide attention and appears to act primarily through its antioxidant and anti-inflammatory mechanism. Studies have shown that in treating oxidation, hypercholesterolemia, inflammation, metabolic syndrome, arthritis, anxiety, and hyperlipidemia, curcumin is useful.

Oxygen is vital to life and can perform biological functions such as catabolism of fat, protein and carbohydrate, and can generate energy for growth, development, and other metabolism. During the normal metabolic function of the body or through the action of chemical substances and ionizing radiations, reactive oxygen species (ROS) is formed and interacts with biomolecules. This ultimately leads to cancer, inflammation, cardiovascular disease (CVD) and other health problems. The reactive oxygen group is a combination of free radicals derived from oxygen (superoxide, hydroxyl radicals, nitric oxide) and highly reactive non-radical derivatives of oxygen (single line oxygen, peroxynitrite, time). The imbalance between free radical formation and removal can lead to oxidative stress-known pathological conditions. The body uses antioxidants to battle these free radicals $[12,13]$.

Curcumin, which is extracted from turmeric by solvent extraction, is the main component of turmeric. Curcumin is stable under conditions of high temperature or acid, but it is unstable under alkaline or light conditions. Curcumin is soluble in oils and bases and at acidic and neutral $\mathrm{pH}$ is nearly insoluble in water. Water-soluble curcumin is prepared by injection into specific micellar surfactant systems (e.g. sodium dodecyl sulfate, cetylpyridinium bromide, gelatin, polysaccharides, polyethylenglycol and cyclodextrins) [14].

Curcumin in dry food is stable. It can be used to heat treated foods and is relatively stable to water. Curcumin has been reported to react with food ingredients by sulfur dioxide bleaching at levels above 100 ppm and formation of complexes with some salts (citrate, phthalate). The main coloring component of curcumin is clearly inactive against chloride, phosphate and bicarbonate. Curcumin's main coloring ingredients are effective food antioxidants. The literature on the active site and the reaction mechanisms involved in antioxidant action is somewhat uncertain and most researchers suggest that antioxidant activity is caused by the moiety of hydroxyl [15].

Research have shown that there is a significant antioxidant effect of curcumin. Curcumin's effect on the prevention of lipid peroxidation is substantially greater than the other spices. The antioxidant activity is 8 times stronger than vitamin $\mathrm{E}$ and more than the artificial antioxidant (BHT) has the effect of preventing the formation of lipid peroxide. Curcumin's antioxidant properties avoid anorexia and provide less fat and free radicals oxidation of foods. Curcumin has good antioxidant properties and does not degrade curry for a long time when holding curry [16].

\section{Processing of turmeric}

The entire raw turmeric (agricultural products) is excavated from the underground. Pure turmeric boiling, drying, fresh color polishing are some of the major post-harvest processing activities being carried out at the farm level. Tilled out Turmeric comprises all the rhizomes of the raw mother and rhizomes of the plant. When the ground fingers are dashed down to the floor, the mother's rhizomes are split. For the convenience of the farmer, the large size rhizomes, subsidiary rhizomes, hands, sora rhizomes and damaged rhizomes are graded and heaped individually [17].

After harvesting, turmeric rhizomes have been found to remove adhering soil, spray residues and other foreign materials. Types of washers are used to soak and spray water. Over night, turmeric is soaked in still water and sprayed over it for cleaning the next day. The next procedure after harvesting is to treat green rhizome. Curing involves boiling fresh water rhizomes and drying them in the sun. This process is also referred to as blanching. Curing gives good color, increases the keeping quality of turmeric and prevent it from deterioration and insects, it also reduces various inconveniences that would be experienced in transporting it to the market. After curing the produce can be stored for a long period. Curing method consists of Boiling, steaming or cooking the fresh rhizomes in water. Boiled rhizomes are dried by the de-hydrator in the sun or artificially. Grinding is a simple cutting process that involves crushing the rhizomes into small particles. The households are buying these bits. In the household stone grinding, 
small pieces of it are grinded. To use Turmeric powder on a large scale, the small pieces of the whole Turmeric pieces must be grinded by machines such as Jowar and Wheat flowering [18].

\section{In vitro studies}

Regular diet contains many antioxidants in our constitution that reduce reverse chemical reactions and trigger cell damage. Agents to reimburse for possibly critical reactive oxygen species observed in organisms. The antioxidant capacity of any substance is defined as the ability to chelate metal ions and to radicalize and retard the action of enzymes in oxidation reactions. Most methods are used to determine a substance's relative antioxidant properties. Antioxidants are reducing agents, and the main antioxidants in vitro are intended primarily to reduce energy incomes. However, it should be clearly recognized that it often experiences certain results such as suppression of singlet oxygen, eradication, in addition to the reduction capacity of its antioxidant substances [19].

Antioxidants are substances obtained in foods by avoiding or interfering with the process of oxidation. Intentionally synthesized antioxidants like BHA, TBHQ and BHT have a broad range of effects in food additives in order to prevent oxidation. Nonetheless, consumer tastes have shifted significantly to organic products with respect to upto-date evidence on possible adverse effects of substances on human health. To raise the concentration is to receive natural antioxidants, to measure carotenoids, vitamin $\mathrm{C}$, tocopherol and phenolic acid, but the most important of these is polyphenols [10].

Consumer preference for natural products has increased in recent years, and the current antioxidant base, particularly spices and herbs, has been highly interested. For the analysis of antioxidant activity, various antioxidant measures such as TPC (total phenol), 1, 1-diphenyl-2-picrylhydrazide (DPCC), $\beta$-carotene, 2,2-azobis,3ethylbenzothiazoline, etc., 6-sulfonic acid, and FRAP (antioxidantreducing fermentation) are used. Turmeric provides a mechanism for inhibiting multiple oxidases, stabilizing the central part of each oxidized lipid, enhancing the antioxidant engineered with different phenolic substances, improving health and related benefits; it is considered to be a specific objective [20].

\section{Functional food and nutraceutics}

As well as the increase in income, consumer demand has changed dramatically in recent years, especially the food manufacturing industry. Today, customers are more aware of food and its role in maintaining health within the human body. Functional nutrition plays a major role in the growth of these foods in this case. Such food is the price of health care, expectations for people's lives are increasing more and the desire of the elder people to live healthy life is increasing more and more to improve the remaining years of life [21].

Functional foods recently contain nutrients such as lipids, carbohydrates, proteins, volatile substances and spices in general. Scientists around the world are engaged in work on functional foods, including genetics, botany, biochemistry, livestock, plant science, nutrition, medicine. Therefore, customers are now deeply interested in such foods and have beneficial effects on health promotion and development, reducing the risk of different diseases. Changing the lifestyles and concerns of people about healthy and nutritious foods encouraged them to choose foods that could promote health and provide essential nutrients. In this case, functional foods and dietary supplements are designed to prevent deceases and improve health, becoming increasingly important throughout the world. The pressure on usable and safe foods for human health treatment has been reduced due to the intervention initiatives. This eventually leads to disease prevention if balanced foods are chosen. The diet of people has changed completely in recent years [22]. In countries around the world, especially in Western countries, different health laws and regulations have been introduced; they concentrate on food quality.

Functional food research has been promoted to tackle physical disorders, but in the area of functional foods further research is needed. With functional foods, the care market has changed to treat multiple chronic diseases. While the functional food field is spreading positively due to the upcoming taste and growing consumer knowledge, more information is needed to educate them about their vital role in growth and development and physiological disorders [23].

Fortification has beneficial effects for human physiology within legal limits. Processed foods, particularly fortified pre-and probiotic foods, enhance physiological function, alleviate many serious diseases, and reduce cholesterol levels and other life-threatening diseases. Intensive demand is increasing rapidly, according to the demographics survey. Revolutionary reforms have been implemented by the Ministry of Health to implement flexible guidelines and services, including the creation of specific health statements for such foods and food products [24].

Similar to other therapeutic drugs, nutraceuticals have no or very low side effect. The term applies to all products, including certain foods such as single nutrients, dietary supplements, herbal products, cereals, juices, soups. Essential nutrients and other health benefits are offered by dietary supplements based on these foods. These foods play an important role in preventing chronic diseases and improving health, greatly prolonging their longevity and delaying the aging process and the body's normal functioning. Plant foods provide not only energy for metabolism but also precursors for protein synthesis and contribute to the use of trace elements [25].

\section{Medicinal foods}

Overall, due to its safe treatment and preventive method, the use of practical and nutritious herbal therapy is growing. Functional foods consist of ingredients that are organic and biologically active. Dietary supplement acceptance is growing, but in most countries, the functional food and nutraceutical market is not widely established. High nutritious goods require careful analysis to determine consumers ' suitability and effect on specific populations. Scientists are increasingly developing functional food development in today's era. Scientists are rapidly improving usable food production in today's era. Innovative food production is numerous undefined activities to proceed with scientific issues, cultural habits, customer satisfaction, age, health, and quantity. In the manufacture of different products, the use of turmeric in various foods is increasing due to its strong therapeutic effect [26].

Egyptian hieroglyphics were among the first full documents that illustrated the use of more than seven hundred herbs or plants as supplements. The Ebers of Papyrus mentioned some of the herbs still in use today, including turmeric [27]. A strong connection existed between food and pharmacology for maintaining health and treating various ailments. Indeed, Hippocrates, $500 \mathrm{BC}$ stated the following: "Let food be your medicine and medicine be your food." For example, spices which normally are used as flavor or taste enhancers in food were described as "influencers of body metabolism". Traditionally used in Indian cooking, turmeric (Curcuma longa) contains the active ingredient curcumin that has antioxidant, anti-inflammatoryand anti- 
carcinogenic properties. This is thought to be regulated by inhibiting several cell signaling pathways, inhibiting enzymes such as cyclooxy genes and S-transferases glutathione, immune regulation, and effects on angiogenesis and cell adhesion. Turmeric has been documented to have LDL cholesterol lowering effects while increasing HDL levels, antihypertensive effects, and caused improved circulation [28]. With rapid progress in pharmacological science, plant-based active ingredients served as model molecules for the future production of new drugs, with aspirin first developed in 1897, derived from salicylic acid. Consequently, this finding led to a period of pharmaceutical development in which synthetic drugs were used to cure most of the ailments. Natural products have acted as a major source of medicines, leading to approximately half of the pharmaceutical armamentarium currently in use. On the other hand, more people are following the selfcare theory and claim that organic foods are associated with fewer side effects and therefore healthier for use. Their health management uses have been the test of time and are often considered relatively cheaper in comparison with synthetic drugs [29]. The past decade has seen an explosion in clinical research demonstrating precisely what health benefits individual foods can provide, identifying the different nutrients and phytochemicals associated with these benefits, and how they can be integrated into the diet. One of the WHO's major challenges was to expand plant research as a potential source of therapy for treating human disease. As an alternative for multi-target therapeutic and phylactic use, rationally formulated poly herbal preparations are slowly being developed. To date, a variety of phytochemicals in medicinal plants has been reported to have a versatile efficacy profile. One sole plant may, for example, contain bitter substances that stimulate digestion, antiinflammatory compounds, polyphenols that can act as antioxidant, and venotonics, antibacterial, and antifungal tannins that perform as natural antibiotics. In certain cases, when a combination of medicinal foods or extracts is consumed at the same time or mixed inappropriate formulation, the therapeutic effects could be a result of total sum of different classes of compounds present within the foods [30]. Yes, it has been stated that the consumption of whole medicinal food has resulted in significantly better outcomes compared to an equivalent dose of single isolated active ingredient. Therefore, synergism can be argued when two or more substances interact in ways that collectively enhance, intensify, or potentiate the influence of each other [31].

\section{Self-modulating disease}

Diet plays an important role in reducing malnutrition and maintaining good health. Simultaneous combination of multiple medical conditions and increased risk of diabetes and cardiovascular disease is characterized as metabolic disorders. Relationship with eating habits is very important; there are possibilities to change living habits and bad eating habits which cause many problems and diseases. Turmeric and their bioactive compounds are also involved to cure cancer, diabetes, cardiovascular complications and immune diseases [32].

Curcumin protects the DNA from single-strand breaks caused by monooxygen. In particular, the mutagenicity of some common mutagens such as tobacco smoke, benzopyrene, DMBA is inhibited by turmeric and curcumin. Curcumin inhibits neutrophil function when administered orally, inhibits platelet aggregation, inhibits lymphocyte involvement, encourages fibrinolysis, and stabilizes the lysosomal membrane. Curcumin also prevents the activation of NF- $\pi \mathrm{B}$, thereby increasing the activation of several inflammatory genes in the downstream. Curcumin prevents aggregation of platelets by inhibiting thromboxane (aggregation promoter) and increasing prostaglandin (inhibiting aggregation of platelets) [33].

Curcumin plays a chemical preventive role by inhibiting first-phase cytochrome p450 enzyme activation of pro-carcinogens; second-phase detoxification function, in particular enhancing glutathione transferase, and inhibiting cell proliferation, angiogenesis, and invasion through various signaling pathways. Curcumin inhibits NF- $\pi \mathrm{B}$ activation and induces down-regulation of specific inflammatory genes [34]. Downregulation of these genes results in signaling for proliferation, antiantigenic activity, and cell invasiveness reduction. Oral administration of turmeric and curcumin extract improves serum lipids, especially total cholesterol, LDL cholesterol, and LDL peroxidation, and increases human HDL cholesterol. Curcumin interferes with intestinal cholesterol intake and increases cholesterol to bile acid by liver cholesterol-7- $\alpha$ hydroxylase (the rate-determining enzyme in bile acid synthesis) and increased bile acid secretion [35].

\section{Hypercholesterolemia}

Hypercholesterolemia is characterized as excessively high levels of plasma cholesterol with elevated cardiovascular disease (CVD) risk factor. Turmeric decreases bad cholesterol (LDL) and raises vitamin $\mathrm{E}$ which functions as an antioxidant, keeping the liver safer by decreasing lipid oxidation and increasing blood cholesterol levels. Hypercholesterolemia happens when there is too much cholesterol in the blood, or high cholesterol. High cholesterol raises the risk of heart disease, stroke, and heart attack. Several studies have shown that turmeric helps to lower the body's high level of cholesterol [36]. In earlier study, Ldlr-mice fed HFD treated with different doses of curcumin $(500,1000$, and $1500 \mathrm{mg} / \mathrm{kg}$ ) decreased plasma lipid concentration resulting in lower lipid accumulation and then reduced fatty acid transport protein expression (CD36/FAT, FABP4/aP2) in peritoneal macrophages. Curcumin in the liver stopped cAMP levels from being suppressed and increased CREB phosphorylation. Curcumin increased the level of cAMP in cell lines, activated the transcription factor CREB and the human promoter CD36 through a sequence containing a CREB response component of consensus. Cholesterol, fatty acid, and triglyceride biosynthesis is followed by regulating gene expression via sterol regulatory element-binding proteins (SREBPs). Cholesterol, fatty acid and triglyceride biosynthesis is followed by controlling gene expression by binding proteins (SREBPs) by sterol regulatory elements. On the other hand, curcumin has shown suppression of SREBP factors and reduction of cholesterol and fatty acid biosynthesis as well as improvement of HFD-induced body weight gain and fat accumulation in the liver, as well as increased serum lipid levels and insulin sensitivity in HFD-induced obese mice [37]. Kim's study results showed that curcumin increased levels of hormonesensitive lipase (HSL) and browning markers (UCP1, PGC-1 $\alpha$ ), which further increased lipolysis and suppressed lipogenesis through transdifferentiation from white adipocytes into brown adipocytes. Kelany reported that curcumin in male Wistar diabetic rats increased the concentration of high density lipoprotein, antioxidant enzyme stages of erythropoietin (EPO), synaptopodin, and connexin 43. Curcumin, in turn, reduced cholesterol, triacylglycerol, phospholipids, $\pi$-glutamyl transpeptidase, MDA, NO, urea, and creatinine. Curcumin showed a significant reduction in desmin, vimentin, SREBP- 1 , TGF- $\beta 1$, and iNOS levels of mRNA expression. Curcumin has been shown to protect the kidneys from sclerosis, glomerular hypertrophy, tubulointerstitial changes, mononuclear cell interstitial infiltration, tubular lipid deposition, and vitro fibroplasia. Cur-NP groups displayed 
Similarly, orally administered curcumin $(200 \mathrm{mg} / \mathrm{kg}$, p.o., once daily) to high-fructose diet (HFD)-induced metabolic syndrome (MetS) rats resulting in a significant reduction in body mass, systolic blood pressure, homeostasis model insulin resistance assessment, glucose concentrations, leptin, total cholesterol, triglycerides, uric acid, MDA, and aTNF- $\alpha$ [38].

Results of Watanabe and Fukui's research showed that curcumin induced major alteration in healthy subjects' serum lipid profile. The TC and HDL-C levels respectively decreased and increased; while the TG serum level remained unchanged. The serum rates of TC, TG, and LDL-Can slightly increased the levels of HDL-C compared to the control group in another long-term daily intake of curcumin (for 30 days). As far as HDL particles are concerned, curcumin has also been shown to induce Apo-A1 expression, which mediates the transfer of cholesterol from cells to HDL particles. Curcumin, on the other hand, was found to increase SR-BI expression in the liver and to modulate hepatocyte removal of HDL-C [39]. The LDL-receptors or LDL-R mediate more than $60 \%$ of LDL clearance. Curcumin has been shown to be effective in inducing various hepatocyte-based LDL-Rs that lead to noticeable reductions in circulation of LDL-C. In contrast, curcumin down controls apoB100's expression as the LDL's main apo lipoprotein. The therapeutic effect of curcumin (CUR) on the serum lipid profile, apolipoprotein A (apo A), apolipoprotein B (apo B), Lipoprotein a Lp (a), homocysteine and endotheline-1 (ET-1) was evaluated in high cholesterol diet-induced hypercholesterolemia in rats in the study conducted by Jang and Choi [40]. Another study on hypercholesterolemic male rats found that a marked reduction in serum total cholesterol, triacylglycerides, LDL-C, VLDL-C, phospholipids, lipoprotein A, Apo B, endothelin-1 and homocysteine was associated with curcumin $(200 \mathrm{mg} / \mathrm{kg}$, body weight/day orally) [41].

\section{Conclusion}

Turmeric has been used to enhance food mood preservation and palatability and improve storage conditions. Researchers and scientists are reported that the mixture of all three is more potent than either one alone. Curcumin has many pharmacological properties such as tumorigenesis inhibition, inflammation, antidepressant, obesity, cardiovascular function, and neurology.

\section{References}

1. Rodrigo R, Miranda A, Vergara L (2011) Modulation of endogenous antioxidant system by wine polyphenols in human disease. J Clinica Chimica Acta 412: 410-424. [Crossref]

2. Quílez J, Ruiz JA, Brufau G, Rafecas M, et al. (2006) Bakery products enriched with phytosterols, $\alpha$-tocopherol and $\beta$-carotene: sensory evaluation and chemical comparison with market products. J Food Chemistry 94: 399-405.

3. Aggarwal BB, Sung B (2009) Pharmacological basis for the role of curcumin in chronic diseases: an age-old spice with modern targets. $J$ Trends in Pharmacological Sciences 30: 85-94.

4. Iniaghe O, Malomo S, Adebayo J (2009) Proximate composition and phytochemical constituents of leaves of some acalypha species. Pakistan Journal of Nutrition 8: 256258 .

5. Okwu D, Josiah C (2006) Evaluation of the chemical composition of two Nigeria medicinal plants. African Journal of Biotechnology 5: 357-361.

6. Pfeiffer E, Hhle S, Solyom A, Metzler M (2003) Studies on the stability of turmeric constituents. J Food Engineer 56: 257-259.

7. Payton F, Sandusky P, Alworth WL (2007) NMR study of the solution structure of curcumin. J Nat Prod 70: 143-146. [Crossref]

8. Peschel D, Koerting R, Nass N (2007) Curcumin induces changes in expression of genes involved in cholesterol homeostasis. J Nutr Biochem 18: 113-119. [Crossref]
9. Hewlings SJ, Kalman DS (2017) Curcumin: A review of its' effects on human health. Foods 18: 167-176. [Crossref]

10. Kunwar A, Priyadarsini KI (2011) Free radicals, oxidative stress and importance of antioxidants in human health. $J$ Med Allied Sciences 1: 53-60.

11. Mudahar GS, Toledo RT, Floros JD, Jen JJ (1989) Optimization of turmeric dehydration process using response surface methodology. Journal of Food Science 54: 714-719.

12. Patil PM, Chhapkhane NK (2013) Improving design and operation of steam based turmeric cooking process. International Journal of Engineering Research and Applications 3: 933-935

13. Iniaghe OM, Malomo SO, Adebayo JO (2013) Proximate composition and phytochemical constituents of leaves of some acalypha species. Pakistan Journal of Nutrition 11: 256-258.

14. Alihosseini F, Azarmi S, Ghaffari S, Haghighat S, Rezayat SSM, et al. (2016) Synergic antibacterial effect of curcumin with ampicillin; free drug solutions in comparison with dsSLN dispersions. J Advanced Pharmaceutical Bulletin 6: 461-465.

15. Kubinarawa D, Ajoku GA, Enwerem N, Okorie DA (2017) Preliminary phytochemical and anti-microbial screening of 50 medicinal plants from Nigeria. African Journal of Biotechnology 6: 1690-1696.

16. Gills LS, Liu CB (2013) Ethno medical uses of plants in Nigeria. J African Press 15: 45-50.

17. Sun YM, Zhang HY, Chen DZ (2002) Theoretical elucidation on the antioxidant mechanism of curcumin: A DFT study. J Org Lett 4: 2909-2911.

18. Reddy ACP, Lokesh BR (1992) Studies on spice principles as antioxidants in the inhibition of lipid peroxidation of rat liver microsomes. J Mol Cell Biochem 111: 117124.

19. Nimse SB, Pal D (2015) Free radicals, natural antioxidants, and their reaction mechanisms. J RSC Advances 5: 27986-28006.

20. Schaffer M, Schaffer PM, Zidan J, Bar Sela G (2011) Curcuma as a functional food in the control of cancer and inflammation. Curr Opin Clin Nutr Metab Care 14: 588-597. [Crossref]

21. Williams M, Pehu E, Ragasa C (2006) Functional foods: opportunities and challenges for developing countries. J Open Nutraceuticals 121: 1061-1065.

22. Schwager J, Mohajeri MH, Fowler A, Weber P (2008) Challenges in discovering bioactives for the food industry. Curr Opin Biotechnol 19: 66-72. [Crossref]

23. Weststrate JA, van Poppel G, Verschuren PM (2002) Functional foods, trends and future. Br J Nutr 88 Suppl 2: S233-235. [Crossref]

24. Allen L, de Benoist B, Dary O, Hurrell R (2006) World health organization, food and agriculture organization. J Guidelines on Food Fortification with Micronutrients 78: 143-146.

25. Keshavarz A, Minaiyan A, Mahzouni P (2013) Effects of Carum carvi L. (Caraway) extract and essential oil on TNBS-induced colitis in rats. J Research in Pharmaceutical Sciences 8: 11-15.

26. Srinivasan K, Bosi S (2005) Spices as influencers of body metabolism: an overview of three decades of research. J Food Research International 38: 77-86.

27. Saunders FR, Wallace HM (2010) On the natural chemoprevention of cancer. J Plant Physiology and Biochemistry 48: 621-626.

28. Dinelli G, Marotti I, Gioia D, Biavati B (2009) Physiologically bioactive compounds of functional foods, herbs, and dietary supplements. J Advances in Food Biochemistry 98: 239-289.

29. Brierley SM, Kelber O (2011) Use of natural products in gastrointestinal therapies. $J$ Current Opinion in Pharmacology 11: 604-611.

30. Gurib-Fakim K, Selim A (2001) Traditional roles and future prospects for medicinal plants in health care. J Asian Biotechnology and Development 13: 77-83.

31. Ncube J, Finnie F, Van Staden J (2012) In vitro antimicrobial synergism within plant extracts combinations from three South African medicinal bulbs. Journal of Ethnopharmacology 139: 81-89.

32. Bengmark S, Mesa MD, Gil A (2009) Plant-derived health: The effects of turmeric and curcuminoids. J Nutricion Hospitalaria 24: 273-281.

33. Srinivas L, Shalini VK, Turmerin (1992) Water soluble antioxidant peptide from turmeric [Curcuma longa]. J Shylaja M Arch Biochem Biophys 292: 617-623. 
34. Srivastava KC, Bordia A, Verma SK (1995) Curcumin, a major component of food spice turmeric (Curcuma longa) inhibits aggregation and alters eicosanoid metabolism in human blood platelets. J Prostaglandins Leukot Essent Fatty Acids 52: 223-227.

35. Soni KB, Kuttan R (1992) Effect of oral curcumin administration on serum peroxides and cholesterol levels in human volunteers. Indian J Physiol Pharmacol 36: 273-275.

36. Chattopadhyan L, Biswas K, Bandyo-Padhyay U, Banerjee RL (2014) Turmeric and curcumin: Biological action and medicinal applications. J Current Science 87: 44-53.

37. Ding L, Li J, Song B, Xiao X, Zhang B, et al. (2016) Curcumin rescues high fat dietinduced obesity and insulin sensitivity in mice through regulating SREBP pathway. Toxicol Appl Pharmacol 304: 99-109. [Crossref]
38. Kelany ME, Hakami TM, Omar AH (2017) Curcumin improves the metabolic syndrome in high-fructose-diet-fed rats: role of TNF- $\alpha, \mathrm{NF}-\mathrm{\kappa B}$, and oxidative stress. Can J Physiol Pharmacol 95: 140-150. [Crossref]

39. Aggarwal YH, Surh S (2007) The molecular targets and therapeutics of curcumin in health and disease. Advances in Experimental Medicine and Biology 58: 270-274.

40. Jang EM, Choi MS, Jung UJ, Kim MJ, Kim HJ, et al. (2008) Beneficial effects of curcumin on hyperlipidemia and insulin resistance in high-fat-fed hamsters. Metabolism 57: 1576-1583. [Crossref]

41. Takashima K, Kohno T, Mori T, Ohtani A, Hirakoso K, et al. (1994) The hypocholesterolemic action of TA-7552 and its effects on cholesterol metabolism in the rat. Atherosclerosis 107: 247-257. [Crossref]

Copyright: @2019 Mughal MH. This is an open-access article distributed under the terms of the Creative Commons Attribution License, which permits unrestricted use, distribution, and reproduction in any medium, provided the original author and source are credited. 